\title{
Zentralvorstandssitzung vom 10. Mai 2012
}

Unterstützung von EQUAM - Die EQUAM-Stiftung soll neu neben den ambulanten Grundversorgern auch Spezialärzte qualitätszertifizieren. Dafür soll EQUAM Qualitätsinstrumente entwickeln. Der Zentralvorstand $(\mathrm{ZV})$ wird EQUAM dabei unterstützen. Er regt an, dass die Stiftung dafür mit interessierten Fachgesellschaften zusammenarbeitet und bei der Umsetzung auch die französischsprachige Schweiz berücksichtigt.

Projekt MARS: Statistiken zur ambulanten Gesundheitsversorgung - Seit dem 1. Januar 2009 gelten neue gesetzliche Bestimmungen bezüglich der Datenlieferungspflicht von Leistungserbringern an die Bundesbehörden. Die FMH moniert am dazu erstellten Grobkonzept MARS das Sammeln von Daten «auf Vorrat» und deren ungenügende bzw. fehlende Anonymisierung, der Bund wird aber MARS unabhängig von unserer Stellungnahme realisieren. Der Zentralvorstand beschliesst, den Aufbau der Statistik kritisch zu begleiten.

Masterplan Hausarztmedizin des EDI - Im Rahmen der Diskussionen um einen indirekten Gegenvorschlag zur Volksinitiative «Ja zur Hausarztmedizin» hat der Bundesrat einen Masterplan zur Prüfung vorgelegt. Dadurch sollen die Anliegen der Hausärztinnen und Hausärzte rascher als über den Verfassungsweg angegangen werden und den Initianten einen Rückzug der Initiative ermöglichen. Innerhalb des
Projekts wurden die Teilprojekte MedBG, Bildung und Forschung sowie Finanzierung und Versorgung gebildet. Der ZV begrüsst das Vorgehen.

Medikamentenverordnung - Der ZV stimmt dem Grundlagenpapier des Ressorts Daten, Demographie und Qualität (DDQ) zur Medikamentenverordnung prinzipiell zu. Es beschreibt diese ärztliche Tätigkeit als komplex und fehleranfällig. Diese Tatsache sollte noch stärker im Bewusstsein der Ärzteschaft verankert werden.

SwissDRG-Version 2.0 - Die FMH nimmt Stellung zur SwissDRG-Version 2.0. Sie schlägt vor, sich in den Workshops der SwissDRG AG für die Implementierung von helvetisierten Zusatzentgelten einzusetzen. So liesse sich das SwissDRG-System trotz ungenügender Datenqualität der Spitäler verbessern. Differenzierte Baserates pro Spitalkategorie sind gemäss FMH keine Dauerlösung zum Ausgleich: Die Systemgüte ist zu verbessern.

Beirat für Ausschaffungsflüge - Die Kommission gegen die Folter möchte, dass die FMH in den Beirat für Ausschaffungsflüge eintritt. Die FMH soll sich dafür einsetzen, dass die Standesordnung und deren Richtlinien für diese Ausschaffungsflüge konsequent umgesetzt werden. Der ZV entspricht diesem Anliegen und wird Mitglied des Beirats. 